\title{
CARACTERÍSTICAS CLÍNICAS E HISTOPATOLÓGICAS ASOCIADAS A PACIENTES CON CÁNCER DE MAMA TRIPLE NEGATIVO EN UN HOSPITAL DE REFERENCIA PERUANO, 2012- 2018
}

\author{
CLINICAL AND HISTOPATHOLOGICAL CHARACTERISTICS ASSOCIATED WITH PATIENTS WITH TRIPLE-NEGATIVE \\ BREAST CANCER AT A NATIONAL REFERENCE HOSPITAL, 2012 - 2018 \\ Giuliana Mandujano-Guizado1,a, Jhony A. De La Cruz-Vargas²,b, William Woolcott-Crispín',c, \\ José Luis Montoya-Suárez ${ }^{3, b}$, Willy Ramos ${ }^{4}$
}

\begin{abstract}
RESUMEN
Objetivo: Determinar las características clínicas e histopatológicas asociadas a las pacientes con cáncer de mama triple negativo (CMTN) en un Hospital peruano de referencia. Métodos: Estudio transversal, analítico. Se revisaron las historias clínicas de todas las pacientes atendidas en el Hospital Nacional Hipóito Unanue durante el periodo de junio del 2012 a junio del 2018. Resultados: Se incluyeron 134 pacientes de los cuales $36(26,9 \%)$ correspondieron a CMTN, el estadío clínico más frecuente fue el III y II para los dos grupos. El tipo histológico que más predomino fue el tipo ductal infiltrante en ambos grupos. El grado histológico elevado fue característico en los casos de CMTN (58\%) a diferencia de los No TN. Los CMTN fueron de mayor tamaño $(5,75 \mathrm{~cm}$ vs $3 \mathrm{~cm}$ en no TN), y el Ki 67 fue más elevado en el TN (35\% en comparación con el No TN con 15\%). Se determinó en el análisis bivariado que el tamaño (RP: 1,4, IC: 1,17-1,68, p=0,0001), Ki 67 (RP: 1,05, IC: 1,03-1,07, $p=0,001$ ), IMC $\geq 25$ kg/m2 (RP: 1,14, IC: 1,23-6,22, $p=0,014$ ) y el Grado Histológico 3 (RP:3,87, IC: $1,74-8,63, p=0,001)$ se asociaron significativamente a la presencia de CMTN. En el análisis multivariado se encontró que el grado 3 (RP: 1,74, IC: 1,01-3, $p=0,0046$ ) y el Ki 67 (RP: 1,02, IC: 1,01-1,03, p=0,0001) fueron las características histopatológicas asociados al CMTN. Conclusión: Las características más consistentemente asociadas al cáncer de mama triple negativo en fueron el elevado grado histológico y mayor valor de Ki67.
\end{abstract}

Palabras clave: Cáncer de mama; Triple negativo; Ki 67. (fuente: DeCS BIREME)

\begin{abstract}
Objective: To determine the clinical and histopathological characteristics associated to patients with triple negative breast cancer of the Hipolito Unanue National Hospital between June 2012 to June 2018. Methods: Cross-sectional, analytical study. We reviewed the medical records of all patients treated at the Hipóito Unanue National Hospital during the period from June 2012 to June 2018. Results: 134 patients were included, out of which 36 (26.9\%) were TN and 98 No TN. Of the histopathological characteristics, the most frequent CD was III and II for the two groups. The histological type that predominated was the infiltrating ductal type in both groups. The high histological grade was characteristic in NT (58\%) unlike non TN. The NTs were larger 5.75 $\mathrm{cm}$ in contrast to the TNs with $3 \mathrm{~cm}$, and the Ki 67 was higher in the TN 35\% in contrast to the NON TN with $15 \%$. In the bivariate analysis the size (RP: 1.4, Cl: 1.17-1.68, $\mathrm{p}=0.0001)$, Ki 67 (RP: 1.05, Cl: 1.03-1.07, $\mathrm{p}=0.001$ ), BMI $\geq 25$ kg / m2 ( RP: 1.14, Cl: 1.23-6.22, p =0.014), Histological Grade 3 (RP: 3.87, Cl: 1.74-8.63, p=0.001), size $\geq 5 \mathrm{~cm}$ (RP: $2.94, \mathrm{Cl}: 1.33-6.48, \mathrm{p}=0.008$ ) were significant. In the multivariate analysis it was found that grade 3 (PR: 1.74, Cl: 1.01-3, p =0.0046) and Ki 67 (RP: 1.02, Cl: 1.01-1.03, $\mathrm{p}=0.0001$ ) were histopathological characteristics associated with CMTN. Conclusion: There are histopathological characteristics associated with triple negative breast cancer particularly high histological grade and Ki67 value.
\end{abstract}

Key words: Breast cancer; Triple negative breast cancer; Ki 67. (source: MeSH NLM)

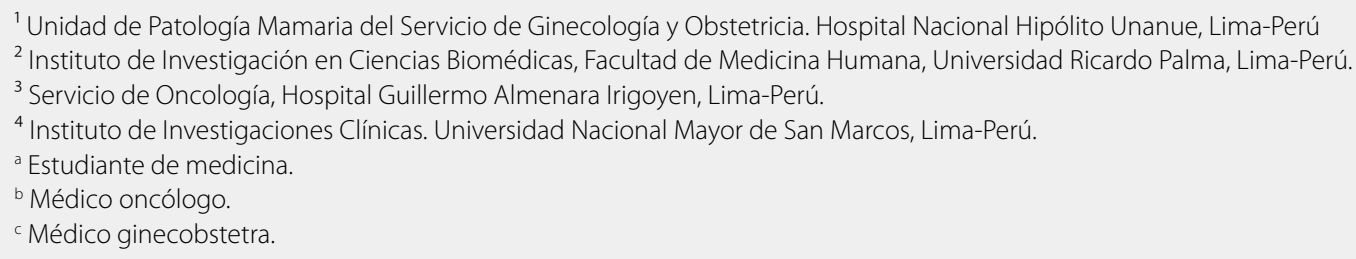

${ }^{1}$ Unidad de Patología Mamaria del Servicio de Ginecología y Obstetricia. Hospital Nacional Hipólito Unanue, Lima-Perú

${ }^{2}$ Instituto de Investigación en Ciencias Biomédicas, Facultad de Medicina Humana, Universidad Ricardo Palma, Lima-Perú.

${ }^{3}$ Servicio de Oncología, Hospital Guillermo Almenara Irigoyen, Lima-Perú.

${ }^{4}$ Instituto de Investigaciones Clínicas. Universidad Nacional Mayor de San Marcos, Lima-Perú.

Estudiante de medicina.

${ }^{\mathrm{b}}$ Médico oncólogo.

Médico ginecobstetra.

Citar como: Giuliana Mandujano-Guizado, Jhony A. De La Cruz-Vargas, William Woolcott-Crispín, José Luis Montoya-Suárez. Características clínicas e histopatológicas asociadas a pacientes con Cáncer de Mama Triple Negativo en un Hospital de Referencia peruano, 2012- 2018. Rev. Fac. Med. Hum. Julio 2019; 19(3):53-59. DOI 10.25176/RFMH.v19i3.2161 


\section{INTRODUCCIÓN}

El cáncer de mama tipo triple negativo es un problema de Salud Pública que va en ascenso debido a su alta incidencia y mortalidad en la población mundial y en especial de Latinoamérica ${ }^{1,2}$.

Ya que este tipo de cáncer de mama al no tener tratamiento sobre blancos específicos, debido a que no presenta receptores de estrógeno y progesterona, y a falta de sobreexpresión del Her-2, no se beneficiaría del tratamiento endocrino y biológico con trastuzumab y sólo respondería a la quimioterapia, siendo el de mejor resultado la quimioterapia neoadyuvante. Debido a esto, en la actualidad es motivo de búsqueda contínua y permanente de investigación para encontrar patrones que ayuden a clasificar su tratamiento o factores de riesgo que mejoren la sobrevida. Se sabe además que el cáncer de mama triple negativo (CMTN) es el más agresivo y con mayor tasa de recurrencia y elevada mortalidad en contraste con los otros subtipos; por lo que resulta importante conocer qué características clínicas e histopatológicas están asociadas en este subtipo de cáncer de mama, para que las pacientes puedan ser tratadas de manera eficiente.Es importante además mencionar que existen pocos estudios del tipo triple negativo de cáncer de mama a nivel nacional.

El objetivo del estudio fue evaluar los factores asociados a la presencia de CMTN en pacientes atendidos en la unidad de patología mamaria del Hospital Nacional Hipólito Unanue durante el periodo 2012 a 2018.

\section{MÉTODOS}

Se realizó un estudio observacional analítico de corte transversal. La población estuvo conformada por los pacientes atendidos en la unidad de cáncer de mama y patología mamaria del servicio de ginecología y obstetricia del Hospital Nacional Hipólito Unanue, durante el período junio 2012- junio 2018, con diagnóstico de cáncer de mama. En cuanto a la muestra, se incluyeron todas las historias clínicas correspondientes al periodo de estudios

Los datos de las pacientes fueron seleccionados de acuerdo a los criterios de inclusión y exclusión.

Se excluyeron aquellos casos con historias clínicas incompletas e ilegibles, pacientes sin diagnóstico histológico ni inmunohistoquímica historias clínicas de pacientes embarazadas, y menores de 18 años.

Para el análisis estadístico, se utilizaron las pruebas de $x^{2}$ de Pearson, considerando como significativo un valor $\mathrm{p}<0,05$; asimismo, se hallaron las razones de prevalencias crudos y ajustados con sus respectivos intervalos de confianza al $95 \%$.

\section{RESULTADOS}

El estudio contó con 134 historias clínicas de pacientes con el diagnóstico de cáncer de mama, de los cuales el $26,9 \%(n=36)$ fueron del tipo Triple negativo y $73,1 \%(n=98)$ No triple negativo (Tabla 1$)$.

Tabla 1. Descripción de los carcinomas mamarios de acuerdo a sus receptores del HNHU junio 2012 a junio 2018.

\begin{tabular}{|c|c|c|c|c|c|c|}
\hline \multicolumn{7}{|c|}{ RECEPTOR } \\
\hline & E & $\mathbf{P}$ & Her 2 & Ki 67 & $\mathbf{n}$ & $\%$ \\
\hline TN & - & - & - & alto & 36 & 26,87 \\
\hline Lum A & + & + & - & $<14 \%$ & 66 & 49,25 \\
\hline Lum B & - & + & - & $\geq 14 \%$ & 3 & 2,24 \\
\hline Lum B & + & - & - & $\geq 14 \%$ & 5 & 3,73 \\
\hline Her 2 & - & - & + & alto & 10 & 7,46 \\
\hline Lum B Her 2 & + & - & + & alto & 6 & 4,48 \\
\hline Lum B Her 2 & + & + & + & alto & 8 & 5,97 \\
\hline
\end{tabular}

La mediana de edad de las pacientes triple negativo (TN) fue de 54,5 $\pm 12,03$ mientras las no TN fue de $52,2 \pm 12,46$ años. $Y$ que las pacientes mayores de 50 años fueron más del grupo No triple negativo (68,3\%) en contraste con los TN $(31,2 \%)$. En el caso del índice de masa corporal (IMC), tanto en el grupo TN con una mediana de IMC de $26,62 \mathrm{~kg} / \mathrm{m} 2$ (DE: 4,31) y No TN con $28,54 \mathrm{~kg} / \mathrm{m} 2$ de IMC (DE: 5,3). Con respecto a los estadío clínicos, en las pacientes con TN fueron más frecuente los estadíos III, II al igual que en los No TN. 
El tipo histológico Ductal infiltrante fue el más frecuente para ambos grupos. El grado histológico 3 fue el más frecuente de los TN a diferencia del grado 2 de los no TN. Acerca del tamaño tumoral, las pacientes TN presentaron tumores de mayor tamaño al momento del diagnóstico 5,75 (Rl: 2-13) $\mathrm{cm}$ vs $3 \mathrm{~cm}(1,2-10)$ del grupo no TN $(p=0,002)$. Además, en el tamaño categorizado, los tumores < $5 \mathrm{~cm}$, fueron en su mayor parte por el grupo No TN $(80,7 \%)$ en comparación con el grupo TN (19,3\%). En cuanto al $\mathrm{Ki} 67$, se halló una diferencia estadísticamente significativa entre ambos grupos, los TN estuvieron representado por un valor promedio de 35\% (RI: 5\%-90\%) en comparación con los No TN con sólo 15\% (Rl: 1\%-90\%) con p=0,0001. En la medición categorizada, el valor de Ki 67 menor de $20 \%$ fue mayor en el grupo no TN (95\%) a diferencia del TN (5\%) al igual que con el valor de Ki67 de 20 a $50 \%$, donde el grupo no TN fue $68,6 \%$ en contraste con el TN (31,8\%). El caso más significativo sucede con el valor de Ki 67 mayor a 50\%, donde los TN son el 74\% en comparación con los no TN con $26 \%$. $(p=0,0001)$. Ver tabla 2.

Tabla 2. Características clínicas e histológicas de las pacientes TN y no TN del HNHU junio 2012 a junio 2018.

\begin{tabular}{|c|c|c|c|}
\hline \multicolumn{4}{|c|}{ cáncer de mama ( $N=134)$} \\
\hline & Triple negativo $N=36(26,9 \%)$ & No triple negativo $\mathrm{N}=98(73,1 \%)$ & Valor P \\
\hline Edad & $54,14 \mathrm{DE}: 12,03$ & $52,20 \mathrm{DE}: 12,46$ & 0,423 \\
\hline Edad categorizada & & & 0,192 \\
\hline$<50$ años & $12(21,05)$ & $45(78,95)$ & \\
\hline$\geq 50$ años & $24(31,17)$ & $53(68,83)$ & \\
\hline IMC & $26,62 \mathrm{DE}: 4,31$ & 28,54 DE: 5,3 & 0,053 \\
\hline IMC & & & 0,012 \\
\hline$\geq 25 \mathrm{~kg} / \mathrm{m} 2$ & $20(20,83)$ & $76(79,17)$ & \\
\hline$<25 \mathrm{~kg} / \mathrm{m} 2$ & $16(42,11)$ & $22(57,89)$ & \\
\hline Estadio clínico & & & 0,045 \\
\hline I & $1(10)$ & $9(90)$ & \\
\hline II & $12(23,53)$ & $39(76,47)$ & \\
\hline III & $18(27,27)$ & $48(72,73)$ & \\
\hline IV & $5(71,43)$ & $2(28,57)$ & \\
\hline Tipo histológico & & & 0,61 \\
\hline CDI & $33(26,83)$ & $90(73,17)$ & \\
\hline $\mathrm{CDL}$ & $3(27,27)$ & $8(72,73)$ & \\
\hline Grado histológico & & & 0,002 \\
\hline 1 & $1(6,67)$ & $14(93,33)$ & \\
\hline 2 & $14(19,44)$ & $58(80,56)$ & \\
\hline 3 & $21(45,65)$ & $25(54,35)$ & \\
\hline Grado Histológico & & & 0,001 \\
\hline $1-2$ & $15(17,24)$ & $72(82,76)$ & \\
\hline 3 & $21(44,68)$ & $26(55,32)$ & \\
\hline Tamaño & 5,75 Rl: 2-13 & 3 RI: 1,2-10 & 0,002 \\
\hline Tamaño categorizado & & & 0,006 \\
\hline$<5 \mathrm{~cm}$ & $17(19,32)$ & $71(80,68)$ & \\
\hline$\geq 5 \mathrm{~cm}$ & $19(41,30)$ & $27(58,70)$ & \\
\hline Ki-67 & 35 RI: 5-90 & $15 \mathrm{Rl}: 1-90$ & 0,0001 \\
\hline Ki-67 categorizado & & & 0,0001 \\
\hline$<20 \%$ & $3(5)$ & $57(95)$ & \\
\hline $20-50 \%$ & $16(31,37)$ & $35(68,63)$ & \\
\hline$>50 \%$ & $17(73,91)$ & $6(26,09)$ & \\
\hline
\end{tabular}


Tabla 3. Tratamiento asignado de cada grupo estudiado.

\begin{tabular}{|c|c|c|c|}
\hline & \multicolumn{3}{|c|}{ Cáncer de mama } \\
\hline & $\begin{array}{c}\text { Triple } \\
\text { negativo } \\
\mathbf{n}=\mathbf{3 6}(26,9 \%)\end{array}$ & $\begin{array}{c}\text { No triple } \\
\text { negativo } \\
n=98 \\
(73,1 \%)\end{array}$ & $\mathbf{p}$ \\
\hline \multicolumn{4}{|l|}{ Tratamiento } \\
\hline 1. Quimioterapia & $36(28.57)$ & $90(71.43)$ & 0.076 \\
\hline $\begin{array}{l}\text { Quimioterapia } \\
\text { neoadyuvante }\end{array}$ & $26(34.21)$ & $50(65.79)$ & 0.028 \\
\hline $\begin{array}{l}\text { Quimioterapia } \\
\text { adyuvante }\end{array}$ & $11(20)$ & $44(80)$ & 0.135 \\
\hline \multicolumn{4}{|l|}{ 2. Tto quirúrgico } \\
\hline $\mathrm{Si}$ & $36(26.87)$ & $98(73.13)$ & \\
\hline No & $2(100)$ & 0 & \\
\hline Tipo de cirugía & & 0.732 & \\
\hline Conservadora & $6(30)$ & $14(70)$ & \\
\hline No conservadora & $28(82.35)$ & $84(17.65)$ & \\
\hline 3. Radioterapia & $22(25)$ & $66(75)$ & 0.317 \\
\hline 4. Hormonoterapia & $1(1.23)$ & $80(98.77)$ & 0.0001 \\
\hline 5. Terapia biológica & 0 & $19(100)$ & 0.002 \\
\hline
\end{tabular}

Todas las pacientes $\mathrm{TN}$ recibieron tratamiento sistémico con quimioterapia $(n=36)$ y la mayor parte de las no TN, además del tratamiento quirúrgico. El tratamiento más frecuente del grupo TN fue la Quimioterapia Neoadyuvante, el esquema más usado en quimioterapia fue AC (adriamicina y ciclofosfamida) con taxanos (paclitaxel), así como también en los no TN $(34,2 \%$ vs $65,8 \% ; p=0,028)$. Seguido de los tratamientos locales, tales como la cirugía, siendo del tipo no conservadora la más frecuente en los TN que en no TN $(82,35 \%$ vs $17,65 \% ; p=0,732)$ y la radioterapia $(p=0,317)$. La hormonoterapia fue el otro tratamiento más frecuente del no TN, además de la quimioterapia y el tratamiento quirúrgico, a diferencia del TN. (98,8\% vs $1,24 \% ; p=0,0001)$. Ver Tabla 3.

De las pacientes TN que recibieron QTn $(n=26)$ sólo 2 ellas lograron respuesta patológica completa (RPC) (7,6\%). Y del grupo no TN que recibieron QTn $(n=50)$ lograron 7 RPC (14\%) con $p=0.29$. Ver tabla 4.

Tabla 4. Respuesta al tratamiento de quimioterapia neoadyuvante de cada grupo estudiado.

\begin{tabular}{|c|c|c|c|}
\hline & \multicolumn{2}{|c|}{ Cáncer de mama } & $\mathbf{P}$ \\
\hline $\begin{array}{l}\text { Respuesta } \\
\text { patológica }\end{array}$ & $\begin{array}{r}\text { Si Triple } \\
\text { negativo }\end{array}$ & $\begin{array}{l}\text { No Triple } \\
\text { negativo }\end{array}$ & 0,295 \\
\hline $\begin{array}{l}\text { Respuesta } \\
\text { completa }\end{array}$ & $2(22,22)$ & $7(77,8)$ & \\
\hline $\begin{array}{c}\text { Respuesta } \\
\text { parcial }\end{array}$ & $19(35,85)$ & $34(64,15)$ & \\
\hline
\end{tabular}

En el análisis bivariado se encontró asociaciones significativas de las siguientes variables, el tamaño del tumor ( $p=0,0001)$, el Ki67 $(p=0,001)$, IMC $(p=0,014)$, grado histológico $(p=0,001)$ y el tamaño categorizado $(p=0,008)$. Con éstas variables significativas se realizó el análisis multivariado.

Tabla 5. Análisis bivariado del CMTN.

\begin{tabular}{|c|c|c|c|}
\hline & \multicolumn{3}{|c|}{ Triple negativo } \\
\hline & $\mathbf{R P}$ & IC(95) & $\mathbf{p}$ \\
\hline Tamaño & 1,4 & $1,17-1,68$ & 0,0001 \\
\hline Ki-67 & 1,05 & $1,03-1,07$ & 0,001 \\
\hline $\begin{array}{l}\mathrm{IMC}(\geq 25 \mathrm{~kg} / \\
\mathrm{m} 2 /<25 \mathrm{~kg} / \mathrm{m} 2)\end{array}$ & 1,14 & $1,23-6,22$ & 0,014 \\
\hline $\begin{array}{l}\text { Grado Histológico } \\
(3 / 1 \text { y } 2)\end{array}$ & 3,87 & $1,74-8,63$ & 0,001 \\
\hline $\begin{array}{l}\text { Tamaño } \\
(\geq 5 \mathrm{~cm} /<5 \mathrm{~cm})\end{array}$ & 2,94 & $1,33-6,48$ & 0,008 \\
\hline
\end{tabular}

Para el análisis multivariado se utilizaron las variables estadísticamente significativas del análisis bivariado que aparecen en la Tabla 5. Se encontró que el grado histológico y el Ki67 están asociados al TN: Aquellas pacientes con grado histológico 3 tuvieron 1,74 veces la probabilidad de tener TN con IC 95\%: 1,01-3; $\mathrm{p}=0,046$, en comparación grado 1,2 ; conforme el Ki 67 aumenta, la probabilidad de TN es 1,02 veces con IC95\%: 1,01-1,03, $p=0,0001$, estando en todos los casos ajustados por las demás variables. (Tabla 6)

Tabla 6. Análisis multivariado del cáncer de mama Triple negativo.

\begin{tabular}{lcrc} 
& \multicolumn{3}{c}{ Triple negativo } \\
& RPa & IC(95) & p \\
\hline $\begin{array}{l}\text { IMC }(\geq 25 \mathrm{~kg} / \\
\left.\mathrm{m}^{2} /<25 \mathrm{~kg} / \mathrm{m}^{2}\right)\end{array}$ & 1,32 & $0,78-2,25$ & 0,301 \\
\hline $\begin{array}{l}\text { Estadio clínico } \\
\text { Grado histológico } \\
(3 / 1 \mathrm{y} 2)\end{array}$ & 1,18 & $0,74-1,87$ & 0,493 \\
\hline $\begin{array}{l}\text { Tamaño } \\
(\geq 5 \mathrm{~cm} /<5 \mathrm{~cm})\end{array}$ & 1,74 & $1,01-3$ & 0,046 \\
\hline $\mathrm{KI} 67$ & 1,13 & $0,62-2,05$ & 0,699 \\
\hline
\end{tabular}

\section{DISCUSIÓN}

El cáncer de mama es una de las neoplasias malignas más frecuentes y causa de mortalidad en mujeres a nivel mundial ${ }^{3,4}$. siendo la primera causa de muerte en mujeres peruanas ${ }^{1,3}$, el tipo Triple negativo tiene una alta incidencia en la población afro latino americana ${ }^{5}$, con una frecuencia de $21,3 \%$ en Perú 4,5 . Además el CMTN presenta alta morbilidad y mortalidad, y no cuenta con tratamiento diana a diferencia de los otros 
tipos de cáncer de mama, siendo solo beneficiario de tratamiento sistémico con quimioterapia ${ }^{1,3}$.

En nuestro estudio, las pacientes con CMTN fueron el $26,9 \%$ de las pacientes con cáncer de mama, cifra similar a lo reportado en la literatura nacional, donde se encontró que los tumores TN fueron el $20-25 \%$. En el 2015, se describe que el 21,3\% de cáncer de mama son TN en el Perú4. El estudio en el año 2015 en Arequipa, donde la prevalencia del CMTN de su estudio fue $30,8 \%^{7}$ y en un estudio en Chiclayo una frecuencia del $32,5 \%$ de $C \mathrm{CTN}^{8}$.

La mediana de edad de las pacientes triple negativo en nuestro estudio fue de 54,14 años, dato similar encontrado un el estudio en Argentina en el 2014 donde la mediana de edad al diagnóstico de TN fue de 54,9 años ${ }^{9}$ y otro estudio en Argentina en 2017 la edad promedio fue de 51 años ${ }^{10}$. Sin embargo estudios nacionales indican una edad más temprana en el diagnóstico de CMTN como se describe en el estudio en el 2015 donde la mediana de presentación es de 48 años, con un $53 \%$ de casos diagnosticados antes de los 50 años ${ }^{4}$. Similar hallazgo se encontró en el estudio, donde la mediana de edad al diagnóstico fue de 40 años en CMTN ${ }^{6}$. La diferencia en cuanto a la edad de diagnóstico encontrada en la literatura con respecto a nuestro estudio puede deberse a varios factores, uno de ellos sería el desconocimiento de la edad para el tamizaje de esta enfermedad o la accesibilidad a los centros hospitalarios para el diagnóstico precoz lo que conllevaría en el retraso del diagnóstico.

En cuanto al IMC, en el estudio nacional realizado en el Instituto Nacional de Enfermedades Neoplásicas, se encontró que de las 89 pacientes que tuvieron CMTN, el $67,4 \%$ tuvieron sobrepeso y obesidad ${ }^{6}$. En otro estudio realizado en nuestro país se describe que el rol del sobrepeso en el CMTN sería controversial al tratarse de un cáncer no hormonal encontrando que las mujeres premenopáusicas y obesas tienen 1.4 veces el riesgo de presentar $\mathrm{CMTN}^{5}$. En el presente estudio se encontró datos similares, ya que la mayor parte de las pacientes tuvieron un IMC $\geq 25 \mathrm{~kg} / \mathrm{m}^{2}$ tanto en el TN $\left(26,6 \mathrm{~kg} / \mathrm{m}^{2}\right.$, DE: 4,31) como en el grupo no TN $\left(28,5 \mathrm{~kg} / \mathrm{m}^{2}, \mathrm{DE}: 5,3\right)$. Asimismo, se encontró que el sobrepeso y obesidad se asociaron a un riesgo $14 \%$ mayor de tener CMTN en comparación que las de bajo y normo peso (RP: 1,14; IC95\%: 1,23-6,22; p: $0,014)$. Sin embargo en un estudio realizado en México menciona que no hubo una relación significativa con el sobrepeso (IMC $\geq 25 \mathrm{~kg} / \mathrm{m} 2 ; \mathrm{p}$ : $0,423)$ u obesidad (IMC $\geq 30 \mathrm{~kg} / \mathrm{m} 2 ; \mathrm{p}: 0,103)^{1}$.

Con respecto al tamaño tumoral se determinó que las pacientes con CMTN tuvieron mayor tamaño al momento del diagnóstico, en el análisis bivariado se encontró que el tamaño tumoral del tipo TN tuvo 5,75 cm (RI: 2-13) a diferencia de los no TN que fue de 3 cm (RI: 1,2-10) con p: 0,002, sin embargo en el análisis multivariado no fue estadísticamente significativo (RP: 1,13; IC95\%:0,62-2,05; p: 0,699). Se conoce que el CMTN está asociado a carcinomas de mayor tamaño a diferencia de los otros tipos tumorales. En un artículo sobre el CMTN en el Perú, se menciona que el promedio del tamaño del tumor fue de $36 \mathrm{~mm}^{4}$. En un estudio argentino el tamaño tumoral encontrado en las pacientes con CMTN fue de $5 \mathrm{~cm}$ al diagnóstico ${ }^{10}$.

El tipo histológico ductal fue el más frecuente tanto en el TN $(91,6 \%)$ como en el No TN (91,8\%).Aunque esta característica no se asoció a la presencia de MMTN la información es consistente con lo reportado en la literatura donde el tipo histológico fue $96,6 \%$ de los casos, carcinoma ductal6. Lo mismo fue reportado en Argentina, donde se encontró que el tipo ductal fue el más frecuente $\mathrm{e}^{9,12-14}$.

Los estadios clínicos fueron otra variable que no fue estadísticamente significativa, s iendo I os e stadios I II y II los más frecuentes en el momento de diagnóstico, tanto en el TN como en el no TN, siendo en los TN el Estadío III y $I I$ el $50 \%$ y $33,3 \%$ respectivamente. Similares hallazgos se encontraron en diversos estudios donde los estadíos clínicos localmente avanzados (II y III) representaron el $95,5 \%$ de los casos $^{6}$. En otro estudio se encontró también mayor incidencia de estadíos III y IV en las pacientes con tumores $\mathrm{TN}^{9} \mathrm{y}$ en otro estudio se encontró mayores casos en estadío II $(49,9 \%)$ y III $(31,1 \%)^{15}$.

La bibliografía internacional reporta que el CMTN está asociado a mayor tamaño y elevado grado histológico, como se ha identificado en nuestro estudio respecto al tamañoy grado histológico III. Se encontró en el análisis multivariado la asociación del grado histológico (3/21) con el CMTN (RP: 1,74, IC95\%: 1,01-3, p: 0,046) y que además el grado histológico 3 fue más frecuente en los TN (58\%) que en el grupo de no TN (26\%). Lo cual concuerda con un estudio nacional, donde el grado histológico III fue el más frecuente, representando el $68,5 \%$ de las pacientes con CMTN${ }^{6}$. Cifras un poco más altas reporta otro artículo, donde el grado histológico 3 fue el más frecuente en el TN comparado con los no TN (73,5\% vs 41,8\%; p: 0,0008) ${ }^{9}$. Y en otro estudio también nos revela que el grado 3 fue el más frecuente, siendo el $74 \%$ de los casos de $\mathrm{TN}^{10}$.

El valor del Ki 67, marcador de proliferación celular y además factor predictivo y pronóstico, fue el que más llamó la atención en el presente estudio, donde 
en el análisis univariado del Ki 67 categorizado, es decir mayor de $50 \%$, se encontró que en las pacientes TN fueron el 74\%, comparado con los no TN siendo sólo $26 \%(p=0,0001)$. Y en el análisis multivariado encontramos significancia estadística donde el valor de Ki 67 se asoció a un incremento del 2\% en la prevalencia por cada incremento en una unidad porcentual del marcador (RP: 1,02; IC95\%: 1,01-1,03; p: 0,0001). Estos hallazgos concuerdan con estudios internacionales, como el del año 2014 en España, donde indican la relación significativa entre el Ki 67, el grado histológico 3 y mayor tamaño tumoral ${ }^{16}$. Diversos estudios señalan la asociación del valor del Ki 67 con el CMTN, como en el estudio (REF) ${ }^{17}$ en el cual se centran en el valor predictivo y pronóstico del Ki 67 en CMTN que recibieron quimioterapia neoadyuvante, donde en el análisis multivariado en las pacientes que no tuvieron RPc tuvo un valor pronóstico independiente para la recaída de la enfermedad (HR: 0,986; IC95\%: 0,978-0,994; p: 0,001), además se indicó que el Ki 67 primario puede ayudar a clasificar en subtipos el CMTN con diferentes respuestas a la quimioterapia ${ }^{17}$ En otro estudio también se identificó la asociación de altos niveles de Ki 67 con el CMTN, además de tener buena respuesta a la quimioterapia neoadyuvante ${ }^{18}$. Al igual en el estudio donde determinan que las pacientes con TN están asociados a niveles altos de Ki 67 ( $\geq 10 \%)$ y además asociada a altas tasas de RPc a la quimioterapia neoadyuvante ${ }^{19}$.

Hace solo dos décadas, el cáncer de mama era considerado una enfermedad simple, con foco especialmente en saber si era estrógenos positivo o no, situación que ha permanecido por más de una centuria.

En tiempos actuales, el cáncer de mama esta caracterizado por su heterogeneidad molecular y clínica.
Se requieren mas estudios base poblacional para disponer de la frecuencia de los subtipos de cáncer de mama en poblaciones latinas e incluso en diversos grupos étnicos.

Finalmente, esta nueva clasificación d el c áncer d e mama triple negativo, presenta una real oportunidad para mejorar los tratamientos y las opciones terapéuticas y arribara una mayor comprensión del rol de los factores del estilo de vida en en la etiologías del cáncer de mama. El cáncer de mama triple negativo representa un reto especial para epidemiilogos, clínicos oncólogos y patólogos.

\section{CONCLUSIÓN}

En conclusión, nuestro estudio muestra que los factores asociados significativamente a la presencia de CMTN fueron el IMC mayor a 25, el tamaño tumoral mayor a $5 \mathrm{~cm}$, el grado histológico y el valor de Ki 67, siendo estos dos últimos los únicos factores asociados en el análisis multivariado.

Contribuciones de autoría: Los autores participaron en la génesis de la idea, diseño de proyecto, recolección e interpretación de datos, análisis de resultados, preparación del manuscrito del presente trabajo de investigación.

Financiamiento: Autofinanciado.

Conflicto de interés: Los autores declaran no tener conflicto de interés en la publicación de este artículo.

Recibido: 12 de marzo 2019

Aprobado: 29 de mayo 2019

Correspondencia: Giuliana Mandujano Guizado.

Dirección: Los Girasoles NCB3, Chaclacayo, Lima-Perú.

Teléfono: 959942996

Correo:Giuliana_2690@hotmail.com 


\section{REFERENCIAS BIBLIOGRÁFICAS}

1. Ramos Muñoz, W. y Venegas Ojeda, D. Análisis de la Situación del cáncer en el Perú, 2013- Lima MINSA Dirección General de Epidemiología, 2013.

2. Oakman C, Viale G, Di Leo A. Management of triple negative breast cáncer. Breast. 2010; 19(5):312-21. doi: 10.1016/j.breast. 2010.03.026.

3. World Health Organization (WHO). GLOBOCAN 20108 Estimated Cancer Incidence, Mortality and Prevalence Worldwide in 2018 [Internet]. International Agency for Research on Cancer. 2018. Disponible en https://www.iarc.fr/wp-content/uploads/2018/09/Globocan_03.jpg (fecha de acceso 23/05/2019)

4. Zaharia M, Gómez H. Epidemiología del Cáncer de mama triple negativo en el Perú. Rev Carcinos. Salud Publica. 2015;5(1):21-25

5. Zaharia M, Gómez H. Cáncer de mama triple negativo: una enfermedad de difícil diagnóstico y tratamiento. Rev Peru Med Exp Salud Publica. 2013;30(4):649-56

6. Neciosup S, Marcelo M, Ventura L, Vallejos C, Gomez H. Factores asociados con la respuesta patológica a la quimioterapia neoadyuvante en el cáncer de mama triple negativo. Carcinos. Salud Publica. 2012;1(1):11-17.

7. Mendoza S, Echegaray A, Caso C. Perfil Inmunohistoquímico del cánce de mama en pacientes de un hospital general de Arequipa, Perú. Rev Med Hered. 2015; 26:31-34

8. Pinto-Larrea IE, Pinto-Tipismana IR. Perfil epidemiológico, clínico y anatomopatológico del cáncer de mama en el hospital naciona Almanzor Aguinaga Asenjo enero-diciembre 2011. Rev Cuerpo Méd HNAAA. 2015;6(1):8-13.

9. Morales SE, Novoa A, Gil L, Bernardi S, MacDonnel M \& Zwenger A. Características clínicas y patológicas del cáncer de mama triple negativo y su comparación con los otros subtipos en pacientes del hospital Provincial Neuquén. Rev Argentina de Mastología. 2014; 33(119):127-137.

10. Orban M, Ulloa A, Arias C, Gon C, Sanchotena V, Carrasco Mary, Horton G, et al. Cáncer de mama Triple Negativo: Evaluación de características clínico-patológicas y factores pronósticos. Revista Argentina de Mastología. 2017; 36(130): 73-86
11. Lara-Medina F, Pérez-Sánchez V, Saavedra-Pérez D, Blake-Cerda M, Arce C, Motorola-Kuba D, et al. Triple Negative Breast Cancer in Hispanic Patients: high Prevalence, por prognosis, and association with menopausal status, body mass index, and parity. Cancer. 2011; 117: 3658-69.

12. Liedtke C,Mazouni Ch, Kenneth R, et al. Response to neoadjuvant Therapy and Long- Term Survival in Patient with Triple Negative Breast Cancer. J Clin Oncol. 2008; 226(8):1275-81

13. Quiróz J, Espinoza K. Supervivencia a 5 años de pacientes con Cáncer de mama Triple Negativo. Asoc Costarricense de Med. 2017; 34(1):1409-15

14. Von Minckwtiz G, Untch M, Blohmer JU, Costa SD, Eidtmann H, Foshing PA, et al. "Definition and Impact of Pathologic Complete Response on Prognosis After Neoadjuvant Chemotherapy in Various Intrinsic Breast Cancer Subtypes. J Clin Oncol. 2012; 30:1796-804.

15. Vallejos CS, Gómez HL, Cruz WR, Pinto JA, Dyer RR, Velarde R, et al. Breast cáncer cla s s if i c a ti o n a c c or d ing t o immunohistochemistry markers: subtypes and association with clinicopathologic variables in a peruvian hospital database. Clin Breast Cáncer. 2010;10(4):294-300. doi: 10.3816/ CBC.2010.n.038.

16. Panal $M$, Herrera $M$, Hardisson $D$, et al. Correlación entre la expresión de Ki67 con factores clásicos pronósticos y predictivos en el cáncer de mama precoz. Rev Senol Patol Mamar. octubre de 2014;27(4):163-9.

17. Wang R, Chen S, Jin Xi \& Shao Zhi. Value of Ki-67 expression in triple negative breast cancer before and after neoadjuvant chemotherapy with weekly paclitaxel plus carboplatin. Scientific Reports Nature. 2016; 6: 30091.

18. Yoshioka T, Hosoda M, Yamamoto M, et al. Prognostic significance of pathologic complete response and Ki67 expression after neoadjuvant chemotherapy in breast cancer. Breast Cancer. 2013. DOI 10.1007/ s12282-013-0474-2.

19. Keam B, Seock-Ah I, Kyung-Hun L, Sae-Won H, Do-Youn O, Jee H, et al. $\mathrm{Ki}-67$ can be used for further classification of triple negative breast cancer into two subtypes with different response and prognosis. Breast Cancer Res. 2011; 13:R22.

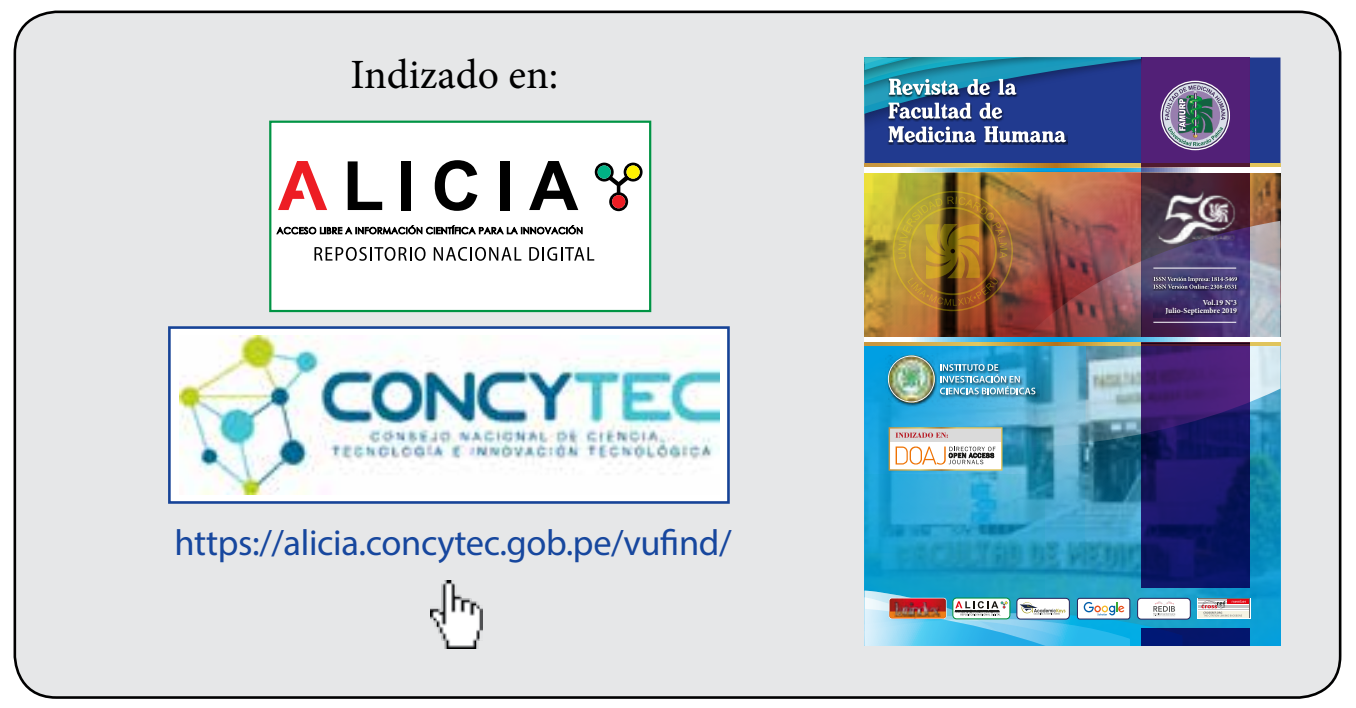

